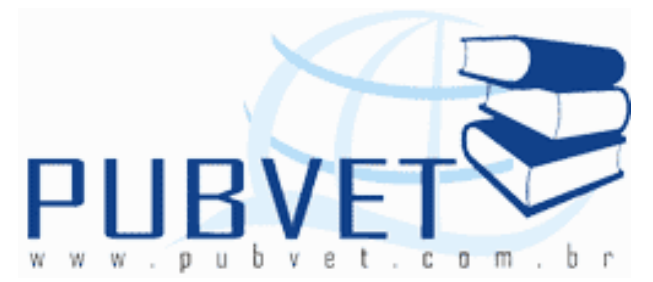

PUBVET, Publicações em Medicina Veterinária e Zootecnia.

\title{
Comportamento anatômico das artérias da base do encéfalo de javali (Sus scrofa scrofa - Linnaeus, 1758)
}

\author{
André Luiz Quagliatto Santos ${ }^{1}$, Lorena Tannús Menezes ${ }^{2}$, Flávio Machado de \\ Moraes $^{3}$, Arthur Paulino Sanzo Kaminishi ${ }^{2}$, Tatiana Grillo Leonardo ${ }^{2}$, Liliane \\ Rangel Nascimento ${ }^{2}$
}

Laboratório de Ensino e Pesquisas em Animais Silvestres - LAPAS, FAMEV/UFU, e-mail: quagliatto@famev.ufu.br 1. Docente. 2. Mestrandos. 3. Professor da Universidade Presidente Antônio Carlos - UNIPAC, Uberlândia.

\section{Resumo}

Visando conhecer mais sobre espécies selvagens do gênero Sus, estudou-se o comportamento das artérias da base do encéfalo de javali (Sus scrofa scrofa) e as modificações no arranjo vascular responsáveis pela irrigação do encéfalo. 0 estudo envolveu 22 espécimes vindos de um criatório de Romaria-MG, através da artéria aorta, foi executada a lavagem do sistema vascular com solução fisiológica, e em seguida se injetou solução de Neoprene Látex "450" corada com pigmento específico, depois disso, foram fixados em solução aquosa de formaldeído a 10\%, por período mínimo de 96 horas, então, os encéfalos foram dissecados e analisados. Com a pesquisa pode-se concluir que as artérias da base do encéfalo estão na dependência da artéria carótida em ambos os antímeros e da artéria basilar e seus ramos; a rede epidural rostral se origina da artéria carótida, das artérias meníngea média e oftálmica 
externa; a artéria carótida apresenta um ramo rostral e um ramo caudal; não existe padrão para as artérias da base do encéfalo deste animal, porém tende seguir o padrão vascular tipo 2 alfa de De Vriese (1905).

Palavras chave: artérias do encéfalo, látex, cérebro, suínos.

\title{
The anatomic behavior of arteries at the base of the brain of wild boar (Sus scrofa scrofa - Linnaeus, 1758)
}

\begin{abstract}
Seeking to know more about wild species of the genus Sus, was studied the behavior of arteries at the base of the brain of wild boar (Sus scrofa scrofa) and changes in the vascular arrangement of the brain responsible for irrigation. This study involved 22 specimens came from a farm in Romaria-MG, and through the aorta was performed a wash of the vascular system with normal saline, and then was injected solution of Neoprene Latex "450" stained with specific pigment, thereafter were fixed in formaldehyde solution to $10 \%$ for at least 96 hours, then the brains were dissected and analyzed. With this research could be concluded that the arteries of the base of the brain are dependent on the carotid artery in both sides and the basilar artery and its branches; the rostral epidural network originates from the carotid artery, the middle meningeal artery and the external ophthalmic artery; the carotid artery shows a rostral and a caudal branch; there is no pattern to the arteries at the base of the brain of this animal, only tends to follow the pattern type 2 alpha of De Vries (1905).
\end{abstract}

Keywords: encephalon arteries, latex, brain, pigs.

\section{INTRODUÇÃO}

O termo javali serve para designar as espécies selvagens do gênero Sus, reservando-se o nome de porco para as raças domésticas. A origem do javali, cuja denominação cientifica é "Sus scrofa", remonta a vários milênios, é 
MENEZES, L.T. et al. Comportamento anatômico das artérias da base do encéfalo de javali (Sus scrofa scrofa - Linnaeus, 1758). PUBVET, Londrina, V. 5, N. 13, Ed. 160, Art. 1085, 2011.

um animal muito antigo, retratado nos desenhos rupestres dos homens das cavernas. O sabor de sua carne, seu tamanho, ferocidade e bravura fizeram do Javali (Sus scrofa scrofa) um dos mais cobiçados troféus de caça, desde a Idade Média. O Javali é o ancestral do porco doméstico, que resultou de vários cruzamentos, acabando por se transformar num animal completamente diferente daquele que o originou. Por causa desse distante parentesco, muitos acham que o javali é o porco da natureza, mas ocorre justamente o contrário. Originário do norte da África e sudoeste da Ásia o javali também migrou para a Europa, onde se disseminou por diversas regiões em função de sua capacidade de adaptação. Na América não é catalogado na fauna nativa; por isso é considerado um animal exótico (LUI, 2000).

Levando em consideração a história evolutiva dos animais e do homem, pode-se dizer que a natureza levou tempo trabalhando com a elaboração do cérebro, e o mesmo certamente ainda será alvo das mais variadas modificações em sua estrutura. De fato, verifica-se um aumento na complexidade na organização do sistema nervoso desde os animais considerados mais primitivos, até aqueles com maior capacidade de exprimir comportamentos mais elaborados (PRADA, 1997).

Considerando as proposições de Tandler (1898), De Vriese (1905), Testut (1911) e Dyce et al. (1990) sobre filogenia e ontogenia do modelo arquitetônico das artérias encefálicas, observa-se que não apenas o encéfalo, mas também o comportamento de seus vasos nas diferentes espécies de mamíferos encontram-se em processo contínuo de modificações, caracterizando-se, a existência de uma relação entre o processo de evolução do sistema nervoso central e as modificações no arranjo vascular responsáveis pela irrigação do órgão.

Com o propósito de pesquisar sobre o arranjo das artérias da base do encéfalo, o anatomista inglês Thomas Willis, em 1664, descreveu detalhadamente a constituição das artérias da base do encéfalo, devido a este fato é conhecido como "círculo ou polígono de Willis" (BARBOSA et al., 1969). 
MENEZES, L.T. et al. Comportamento anatômico das artérias da base do encéfalo de javali (Sus scrofa scrofa - Linnaeus, 1758). PUBVET, Londrina, V. 5, N. 13, Ed. 160, Art. 1085, 2011.

Muitos pesquisadores mostraram-se interessados na variedade anatômica desses vasos, tendo sido muitos os trabalhos empreendidos com o objetivo de melhor conhecer o seu comportamento. Mais recentemente, têm sido evidenciadas variações relacionadas à vascularização arterial do encéfalo entre diferentes espécies animai: (FERREIRA, J. e PRADA, I., 2009) em primatas não humanos, (SANTOS et al., 1993; CAMPOS et al., 2003) em eqüinos da raça Puro Sangue Inglês, (ALCÂNTARA, 1992) em cães sem raça definida, (SANTOS et al., 1993) em ovinos, (LINDERMANN, 1994) em gambá, (RECKZIEGEL, 1994) em capivara, (MELO, 1996) em bovinos azebuados, (FERREIRA, J., 1997) em macaco prego, (FERREIRA, C., 1998; FERREIRA, J. e PRADA, I., 2005; CAMARA FILHO et al., 2005) em suínos, (LIMA et al., 2005) em suínos da linhagem Camborough 22.

Desenvolveu-se tema similar, relativo ao javali, devido ao aumento do número de criatórios e a excelente qualidade nutricional da carne deste animal, quando comparada a outros animais que são destinados ao consumo (em uma porção de $100 \mathrm{~g}$ temos: Javali: $160 \mathrm{cal}, 2,8 \mathrm{~g} /$ gordura, 45 mg/colesterol; Porco: 219 cal, 10,64 g/gordura, $101 \mathrm{mg} /$ colesterol; Bovino: $214 \mathrm{cal}, 9,76 \mathrm{~g} /$ gordura, $92 \mathrm{mg} /$ colesterol) (LUI, 2000).

Com o estudo do comportamento das artérias da base do encéfalo de javali (Sus scrofa scrofa), obteve-se informações referentes aos aspectos neuromorfológicos deste animal, oferecendo subsídios que venham diminuir a escassez de dados na literatura em termos comparativos.

\section{MATERIAL E MÉTODOS}

Foram utilizados 22 exemplares de javalis (Sus scrofa scrofa-Linnaeus, 1758), 14 fêmeas e 08 machos, procedentes de um criatório (autorizado pelo IBAMA) situado no município de Romaria-MG.

Os animais foram encaminhados ao Laboratório de Pesquisa em Animais Silvestres (LAPAS) da Faculdade de Medicina Veterinária da Universidade Federal de Uberlândia. Em 20 animais procedeu-se a dissecação na região 
MENEZES, L.T. et al. Comportamento anatômico das artérias da base do encéfalo de javali (Sus scrofa scrofa - Linnaeus, 1758). PUBVET, Londrina, V. 5, N. 13, Ed. 160, Art. 1085, 2011.

torácica (entre o sétimo e oitavo espaço intercostal), individualizou-se a artéria aorta e com auxílio de cânula de calibre compatível com o diâmetro do vaso, procedeu-se a lavagem do sistema vascular com solução fisiológica e, em seguida, injetou-se solução de Neoprene Látex "450" (Du Pont do Brasil S.A. Indústrias Químicas) corada com pigmento específico (Globo S.A.-Tintas e Pigmentos). Logo após, os animais foram fixados em solução aquosa de formaldeído à $10 \%$, por período mínimo de 96 horas, na qual ficaram armazenados. Os encéfalos foram dissecados, removendo-se pele e musculatura anexa aos ossos do crânio, posteriormente foram abertas as caixas cranianas, e retiraram-se os encéfalos.

Buscando a visualização das artérias destinadas ao encéfalo, no tocante aquelas formadoras das redes admiráveis epidurais rostrais, confeccionaram modelos em Acetato de Vinyl (VMCHB - 1099 Union Carbide Corporation Chemical and Plastic N.Y. - USA) das artérias cranianas, sendo utilizados para a fabricação dois javalis machos. Promoveu, no antímero esquerdo, uma abertura longitudinal da cavidade torácica, ao nível do quinto espaço intercostal. Em seguida, o tronco braquioencefálico foi isolado, sendo introduzido em sua luz, em sentido cranial, cânula com calibre compatível ao diâmetro do vaso. O sistema arterial na região de pescoço e cabeça receberam injeção de acetona P.A. (Merck S/A Indústria Química - Rio de Janeiro- RJ), e posteriormente solução corada de Acetato de Vinyl. Em seguida, esperou-se 24 horas para realização de um corte transversal na região cervical (entre a quarta e quinta vertebras cervicais), separando a cabeça do corpo do animal. Após este procedimento as cabeças foram imersas em uma solução aquosa de Ácido Sulfúrico 30\% (P.A.-A.C.S. Nuclear) permanecendo aproximadamente 168 horas nesta solução.

Durante a dissecação foram confeccionadas fotografias e anotações da origem, o trajeto e a área de destino das artérias da base do encéfalo.

Os termos utilizados para a designação dos vasos arteriais estudados, estão de maneira geral de acordo com o International Committee on Veterinary Gross Anatomical Nomenclature (2005). 
Em contrapartida, utilizaram-se, à semelhança de Ferreira, C. e Prada, I. (2005) e Lima et al. (2005), as denominações "circuito arterial" do encéfalo, "ramo rostral da artéria carótida do encéfalo", "ramo caudal da artéria carótida do encéfalo", no que tange ao comportamento das artérias da base do encéfalo de javali (Sus scrofa scrofa).

\section{RESULTADOS}

De acordo com o enfoque proposto para o comportamento das artérias da base do encéfalo de javali (Sus scrofa scrofa) estiveram expostas as artérias originárias do circuito arterial do encéfalo e seus arranjos morfológicos.

A origem e disposição das artérias que se destinam a base do encéfalo serão expostas conforme os itens relacionados.

\subsection{ORIGEM DAS ARTÉRIAS DA BASE DO ENCÉFALO}

4.2. DISPOSIÇÃO DAS ARTÉRIAS DA BASE DO ENCÉFALO (Figuras 1,2 e 3):

4.2.1. ARTÉRIA CARÓTIDA DO ENCÉFALO (Figura 1)

4.2.2. RAMO ROSTRAL DA ARTÉRIA CARÓtIDA DO ENCÉFALO (Figura 1):
4.2.2.1. ARTÉRIA CORÓIDEA ROSTRAL (Figura 1)
4.2.2.2. ARTÉRIA CEREBRAL MÉDIA (Figura 2)
4.2.2.3. ARTÉRIA CEREBRAL ROSTRAL (Figura 2)
4.2.2.4. ARTÉRIA ETMOIDAL INTERNA (Figura 2)
4.2.2.5. ARTÉRIA COMUNICANTE ROSTRAL (Figura 1)

\subsubsection{RAMO CAUDAL DA ARTÉRIA CARÓtIDA DO ENCÉFALO}

(Figura 1):
4.2.3.1. ARTÉRIA CORÓIDEA CAUDAL (Figura 1)
4.2.3.2. ARTÉRIA CEREBRAL CAUDAL (Figura 1) 
4.2.4. ARTÉRIA BASILAR (Figura 3):

4.2.4.1. ARTÉRIAS PARA O BULBO (Figura 3)

4.2.4.2. ARTÉRIAS PARA A PONTE (Figura 3)

4.2.4.3. ARTÉRIA CEREBELAR CAUDAL (Figura 3)

4.2.4.4. ARTÉRIA CEREBELAR ROSTRAL (Figura 1)

4.2.5. ARTÉRIAS MESENCEFÁLICAS (Figura 1)

4.2.6. CIRCUITO ARTERIAL DO ENCÉFALO (Figura 1, 2 e 3)

\subsection{ORIGEM DAS ARTÉRIAS DA BASE DO ENCÉFALO:}

As artérias carótida interna, artéria maxilar interna e seus ramos (meníngea média, oftálmica externa) e occipital originam as artérias da base do encéfalo de javali, em ambos os antímeros.

A artéria vertebral em ambos os antímeros, anastomosa-se com a artéria occipital onde penetra no forame vertebral lateral, constituindo com a homônima do lado oposto, na região entre a medula espinhal e o bulbo, a artéria basilar rostralmente e a artéria espinhal ventral caudalmente.

A artéria carótida interna juntamente com a artéria maxilar interna e seus ramos participam da formação da rede admirável epidural rostral, onde a partir desta rede tem-se a emergência da artéria carótida do encéfalo com seus ramos; ramo rostral da artéria carótida do encéfalo e ramo caudal da artéria carótida do encéfalo.

\subsection{DISPOSIÇÃO DAS ARTÉRIAS DA BASE DO ENCÉFALO (Figuras 1,2 e 3):}

\subsubsection{ARTÉRIA CARÓtIDA DO ENCÉFALO (Figura 1)}

A artéria carótida do encéfalo tanto no antímero direito como no esquerdo, possui um curto trajeto se originando na rede admirável epidural 
MENEZES, L.T. et al. Comportamento anatômico das artérias da base do encéfalo de javali (Sus scrofa scrofa - Linnaeus, 1758). PUBVET, Londrina, V. 5, N. 13, Ed. 160, Art. 1085, 2011.

rostral, sendo dividida em um ramo rostral e um ramo caudal. A artéria carótida do encéfalo foi evidenciada em $100 \%$ dos modelos encefálicos.

\subsubsection{RAMO ROSTRAL DA ARTÉRIA CARÓtIDA DO ENCÉFALO (Figura1):}

O presente ramo representa a divisão terminal rostral da artéria carótida do encéfalo, em todos os modelos. Caminhando rostralmente e medianamente em direção à fissura longitudinal do cérebro. Emite em ambos os antímeros a artéria coroídea rostral, artéria cerebral média e a artéria cerebral rostral, sendo esta após a última artéria cerebral média (sentido caudorostral) em ambos os antímeros.

A artéria cerebral rostral esquerda em um modelo (5\%) como já exposto, originou-se antes da última (sentido caudorostral) artéria cerebral média.

Em relação ao calibre, tanto o ramo rostral da artéria carótida do encéfalo quanto o ramo caudal da artéria carótida do encéfalo mostraram-se semelhantes sem grandes variações, porém os ramos rostrais apresentaramse com um pequeno aumento no calibre quando da sua origem, em relação ao ramo caudal.

\subsubsection{ARTÉRIA COROÍDEA ROSTRAL (Figura 1)}

A artéria coroídea rostral em ambos os antímeros, mostraram-se simetria em seu comportamento, ou seja, aprofundaram-se em relação ao lobo piriforme ( $100 \%$ dos modelos).

A artéria coroídea rostral no antímero direito em 19 modelos (95\%), tiveram origem no ramo rostral da artéria carótida do encéfalo, o mesmo aconteceu no antímero esquerdo, porém em 17 modelos (85\%).

Em um caso a artéria em questão do antímero esquerdo (5\%), apresentou-se originária tanto do ramo rostral da artéria carótida do encéfalo 
quanto do ramo caudal da artéria carótida do encéfalo. Unindo-se logo após sua origem, formando um vaso único.

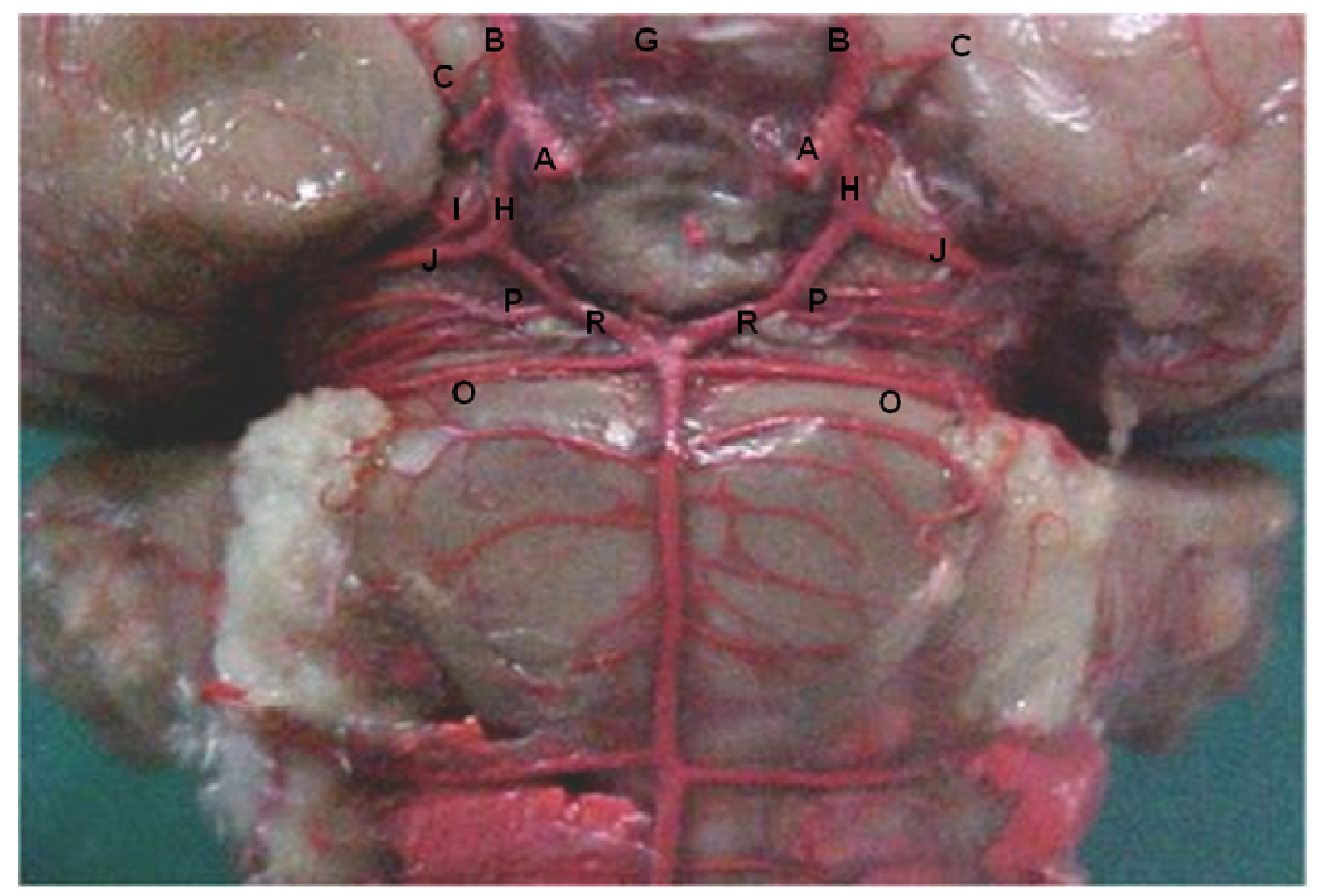

Figura 1: Vista ventral do encéfalo Sus scrofa scrofa fêmea, apresentando o Circuito Arterial e suas artérias. A: artéria carótida do encéfalo; B: ramo rostral da artéria carótida do encéfalo; C: artéria coróidea rostral; G: artéria comunicante rostral; H: Ramo caudal da artéria carótida do encéfalo; I: artéria coróidea caudal; J: artéria cerebral caudal; O: artéria cerebelar rostral; $\mathrm{P}$ : artéria mesencefálica; $\mathrm{R}$ : ramo terminal da artéria basilar.

\subsubsection{ARTÉRIA CEREBRAL MÉDIA (Figura 2)}

A artéria cerebral média em ambos os antímeros, tem sua origem no ramo rostral da artéria carótida do encéfalo (100\%). Sendo representado geralmente por dois a cinco vasos arteriais que se dirigiram laterodorsalmente atingindo a região do trígono olfatório assim como o sulco rinal lateral, preenchendo, no entanto a fissura transversa do cérebro e distribuindo nas 
MENEZES, L.T. et al. Comportamento anatômico das artérias da base do encéfalo de javali (Sus scrofa scrofa - Linnaeus, 1758). PUBVET, Londrina, V. 5, N. 13, Ed. 160, Art. 1085, 2011.

porções lateral, dorsolateral, caudolateral e rostrolateral do hemisfério cerebral.

No tocante ao número de vasos arteriais relativos a artéria cerebral média, obtiveram no antímero direito em dois modelos (10\%) a presença de duas artérias, em 13 modelos (65\%) a presença de três artérias, em quatro modelos $(20 \%)$ a presença de quatro artérias e em um modelo (5\%) a presença de cinco artérias. Já no antímero esquerdo encontraram-se quatro modelos $(20 \%)$ a presença de duas artérias, em nove modelos $(45 \%)$ a existência de três artérias, em seis modelos (30\%) a presença de quatro artérias e em um modelo ( $5 \%)$ a presença de cinco artérias.

\subsubsection{ARTÉRIA CEREBRAL ROSTRAL (Figura 2)}

A artéria cerebral rostral em ambos os antímeros, nos 20 modelos (100\%) apresentou-se como divisão terminal do ramo rostral da artéria carótida do encéfalo, tendo aquela emergência a partir de um único vaso, em cada antímero, em todos os animais. Percorre rostralmente no plano sagital mediano ao longo da fissura longitudinal do cérebro.

\subsection{ARTÉRIA ETMOIDAL INTERNA (Figura 2):}

A artéria etmoidal interna continua rostralmente margeando a fissura longitudinal do cérebro, em direção à parte rostral do bulbo olfatório onde emite ramos para esta estrutura, atingindo a lâmina crivosa do etmóide, onde forma a rede etmoidal. 




Figura 2: Vista ventral do encéfalo Sus scrofa scrofa fêmea, apresentando o Circuito Arterial e suas artérias. D: artéria cerebral média; E: artéria cerebral rostral; F: artéria etmoidal interna.

\subsubsection{ARTÉRIA COMUNICANTE ROSTRAL (Figura 1)}

A artéria comunicante rostral localiza-se rostralmente ao quiasma óptico, mostrando um tracto anastomótico de disposição transversal (ambos os antímeros) entre as artérias cerebrais médias, promovendo o fechamento do circuito arterial do encéfalo rostralmente $(100 \%)$, sendo representada em oito modelos $(40 \%)$ por um único vaso (antímero esquerdo e direito); em quatro situações (20\%) representadas por dois vasos arteriais (em ambos antímeros); e em oito modelos (40\%) por uma formação em rede.

\subsubsection{RAMO CAUDAL DA ARTÉRIA CARÓTIDA DO ENCÉFALO (Figura 1):}

As artérias carótidas do encéfalo em ambos os antímeros deram origem aos ramos caudais em todos os modelos (100\%). Dirigi-se caudamente 
MENEZES, L.T. et al. Comportamento anatômico das artérias da base do encéfalo de javali (Sus scrofa scrofa - Linnaeus, 1758). PUBVET, Londrina, V. 5, N. 13, Ed. 160, Art. 1085, 2011.

unindo-se com os ramos terminais da artéria basilar. Conforme já exposto o calibre do ramo caudal da artéria carótida do encéfalo e o calibre do ramo rostral da artéria carótida do encéfalo são semelhantes, não mostrando grandes variações.

\subsubsection{ARTÉRIA COROÍDEA CAUDAL (Figura 1)}

A partir do ramo caudal da artéria carótida do encéfalo, teve-se origem a artéria coroídea caudal, em dois modelos no antímero esquerdo (10\%), e em uma modelo (5\%) no antímero direito.

\subsubsection{ARTÉRIA CEREBRAL CAUDAL (Figura 1)}

As artérias cerebrais caudais que emergiram dos ramos caudais das artérias carótida do encéfalo nas 20 modelos (100\%), após suas emergências dirigiram-se laterodorsalmente às porções caudais dos hemisférios cerebrais.

Relativamente a origem da artéria cerebral caudal, no ponto de união entre o ramo caudal da artéria carótida do encéfalo e o ramo terminal da artéria basilar, temos que no antímero direito em três modelos (15\%) a artéria cerebral caudal originou-se do ramo terminal da artéria basilar, em 17 casos (85\%) a artéria em questão originou-se do ramo caudal da artéria carótida do encéfalo. Já no antímero esquerdo temos a seguinte situação; em quatro modelos $(20 \%)$ a artéria cerebral caudal emergiu do ramo terminal da artéria basilar e em 16 modelos (80\%) a artéria cerebral caudal se originou do ramo caudal da artéria carótida do encéfalo.

\subsubsection{ARTÉRIA BASILAR (Figura 3):}

A artéria basilar é um vaso ímpar resultante da união das artérias occipitais esquerda e direita no terço caudal do bulbo, próximo da transição deste com a medula espinhal, localizam-se ventralmente a ponte e bulbo. Esta 
artéria mostrou uma diminuição de calibre de forma significativa a partir da artéria cerebelar caudal, até a origem da artéria cerebelar rostral (100\%). Neste momento, se considerarmos o direcionamento da artéria basilar em direção ao mesencéfalo (antímero esquerdo e direito) teremos a presença de ramos terminais em ambos antímeros, podendo estes ramos originarem a artéria cerebral caudal. Porém se tomarmos como nota o calibre da artéria basilar, não teria a presença dos ramos terminais, sendo estes segmentos originários do ramo caudal da artéria carótida do encéfalo.

\subsubsection{ARTÉRIAS PARA O BULBO (Figura 3)}

Os ramos da artéria basilar destinadas para o bulbo mostraram-se de menor calibre comparadas com a originária destes ramos. Surgiram-se caudalmente à emergência da artéria cerebelar caudal, mostrando certa simetria de modo geral em sua distribuição nos dois antímeros.

\subsubsection{ARTÉRIAS PARA A PONTE (Figura 3)}

Os ramos da artéria basilar para a ponte em ambos os antímeros são delgados, dirigindo-se lateralmente sobre a ponte e distribuindo-se de forma geralmente simétrica em relação à origem destes ramos.

\subsubsection{ARTÉRIA CEREBELAR CAUDAL (Figura 3)}

Em se tratando da artéria cerebelar caudal temos que em todos os modelos tanto a esquerda como a direita, elas mostraram-se representadas por um único vaso.

As artérias cerebelares caudais esquerda (95\%) e direita (90\%) em ambos os antímeros originaram-se tendo certa simetria em relação ao terço médio da artéria basilar na face ventral do bulbo. A artéria cerebelar caudal esquerda em relação a sua origem mostrou-se em uma posição mais caudal 
em relação a transição da ponte com o bulbo (5\%), a artéria cerebelar caudal direita (5\%) apresentou-se também mais caudalmente em relação ao mesmo referencial citado acima, já a artéria cerebelar caudal direita (5\%) encontrouse em posição mais rostral, próximo da artéria cerebelar rostral, ou seja, na face ventral da ponte, mostrando assimetria no tocante a sua emergência.

A artéria cerebelar caudal em ambos os antímeros tiveram uma direção em sentido laterocaudal e distribuíram-se nas porções caudais dos hemisférios cerebelares. A partir da artéria cerebelar caudal surgiu-se um ramo rostrolateral em ambos antímeros, que se destinaram ao terceiro par de nervos cranianos, à este ramo denominou-se de artéria cerebelar média esquerda e a artéria cerebelar média direita. (100\%).



Figura 3: Vista ventral do encéfalo Sus scrofa scrofa fêmea, apresentando o Circuito Arterial e suas artérias. K: artéria basilar; L: ramos para o bulbo; M: ramos para ponte; $\mathrm{N}$ : artéria cerebelar caudal; S: artéria occipital; T: artéria espinhal ventral. 
MENEZES, L.T. et al. Comportamento anatômico das artérias da base do encéfalo de javali (Sus scrofa scrofa - Linnaeus, 1758). PUBVET, Londrina, V. 5, N. 13, Ed. 160, Art. 1085, 2011.

\subsubsection{ARTÉRIA CEREBELAR ROSTRAL (Figura 1)}

As artérias cerebelares rostrais esquerda e direita mostraram-se como vasos únicos em ambos os antímeros; dirigiram-se laterodorsalmente acompanhando a porção rostral da ponte, se ramificando para as porções rostrais do cerebelo.

Em relação a origem tivemos que no antímero direito, em 11 modelos (55\%) a artéria cerebelar rostral emergiu do ramo terminal da artéria basilar, em nove modelos (45\%) a artéria cerebelar rostral teve origem na artéria basilar. No antímero esquerdo, em 10 modelos (50\%) emergiu do ramo terminal da artéria basilar, em 10 modelos (50\%) a artéria cerebelar rostral teve origem na artéria basilar.

\subsubsection{ARTÉRIAS MESENCEFÁLICAS (Figura 1):}

As artérias mesencefálicas possuem trajeto em direção à porção dorsal do mesencéfalo, sendo representadas por número variável de vasos arteriais e localizando-se sempre, sua origem, entre a artéria cerebral caudal e a artéria cerebelar rostral, ou seja, no ramo terminal da artéria basilar.

Apresentou-se em seis modelos (30\%) no antímero direito com um vaso arterial, em 10 modelos (50\%) com duas artérias, em quatro modelos (20\%) com três artérias mesensefálicas.

No antímero esquerdo mostrou-se com seis modelos (30\%) com um único vaso, em 12 modelos (60\%) com vaso duplo, em um modelo (5\%) três vasos e em um modelo ( $5 \%$ ) com quatro artérias.

Em um modelo (5\%) no antímero direito encontramos anastomose da única artéria mesencefálica, entre o ramo terminal da basilar com a artéria cerebelar rostral

Evidenciamos também em um modelo ( $5 \%$ ) no antímero esquerdo, a presença de anastomose em uma das artérias mesencefálica, localizada entre a artéria cerebral caudal e o ramo terminal da artéria basilar. 


\subsubsection{CIRCUITO ARTERIAL DO ENCÉfALO (Figuras 1, 2 e 3):}

Em relação a disposição apresentada pelo circuito arterial do encéfalo de javali (Sus scrofa scrofa) pode-se notar que o mesmo delimitou o corpo mamilar, o túber cinério, a glândula hipófise e o quiasma óptico em todos os modelos encefálicos.

O circuito arterial do encéfalo em todos os modelos (100\%) mostrou-se representados pelos ramos rostrais e caudais das artérias carótidas do encéfalo em ambos antímeros.

Os ramos rostrais das artérias carótidas do encéfalo formaram a porção rostral desta estrutura e dirigiram-se em direção lateral, e após cruzarem os tractos ópticos constituíram um arco de concavidade medial, isto foi evidenciado em todos os modelos. O circuito arterial do encéfalo apresentou-se fechado rostralmente $(100 \%)$ devido a formação das artérias comunicantes rostrais

Em relação a porção caudal do circuito arterial do encéfalo, notaram-se a existência de ramos caudais das artérias carótidas do encéfalo e ramos terminais da artéria basilar de cada antímero, estes vasos limitaram caudalmente o corpo mamilar o túber cinério e a glândula hipófise.

Todos os animais investigados, tiveram a porção caudal do circuito arterial do encéfalo semelhante a metade caudal de um polígono.

\section{DISCUSSÃO}

Tomando como partida o suprimento sangüíneo para o encéfalo, verificamos em relação ao comportamento das artérias da base do encéfalo de javali, que este arranjo se encontra dependente da artéria carótida comum, artéria maxilar interna, artéria meníngea média, artéria oftálmica externa e artéria occipital, assim como Flechsig e Zintzsch (1969); comenta que a rede admirável epidural em suínos é formada por ramos das artérias carótida interna, meníngea média, maxilar interna e oftálmica sendo também 
MENEZES, L.T. et al. Comportamento anatômico das artérias da base do encéfalo de javali (Sus scrofa scrofa - Linnaeus, 1758). PUBVET, Londrina, V. 5, N. 13, Ed. 160, Art. 1085, 2011.

responsável pelo suprimento do encéfalo. Tendo a partir deste, a origem da artéria basilar que juntamente com os ramos rostral e caudal da artéria carótida do encéfalo, constituem o circuito arterial do encéfalo do espécime em questão.

Concordando com as exposições de Bruni e Zimmerl, 1947; Gonzalez Y Garcia e Gonzalez Alvarez, 1929; Mannu, 1930; Montané e Bourdelle, 1920; Nanda, 1986; Schwarze e Schröder, 1972; Simoens et al., 1987; Scremin, 1995 no tocante a origem da rede admirável epidural rostral, tendo esta rede no Sus scrofa scrofa também origem na artéria carótida interna, artéria maxilar interna, artéria meníngea média e artéria oftálmica externa.

Várias colocações foram denominadas por diversos autores, rete mirabile por Baptista, 1922; Mannu, 1930; Montané e Bourdelle, 1920, "de rede admirável epidural Bruni e Zimmerl, 1947; Ellenberger e Baum, 1943; Gonzalez Y Garcia e Gonzalez Alvarez, 1929 e de "rete mirabile caroticum" Crosby e Schnitzlein, 1982, todas as denominações acima para a rede em questão traduzem bem a estrutura da rede admirável e que podem ser aplicadas para o javali.

Nos modelos realizadas foram encontradas, assim como Gillilan (1974, 1984) a presença de uma desenvolvida rede admirável.

Indo ao encontro das preposições de Flechsig e Zintzsch (1969), os animais investigados também demonstram as artérias meníngea média e oftálmica externa cedendo ramos para a rede epidural rostral.

Ainda em relação a rede admirável epidural rostral temos que a presente pesquisa concorda com as alusões referidas por Câmara Filho et al. (2004), Ferreira, C. (1998), Lima et al. (2005) feitas nos suínos sobre a artéria carótida do encéfalo que provém da rede admirável epidural rostral, por sua vez constituída com a participação das artérias carótida interna e maxilar com seus ramos, as artérias meníngeas média e oftálmica externa, caracterizando a participação da artéria carótida interna.

No tocante a artéria carótida interna, observamos sua origem em tronco comum com a artéria occipital, logo a seguir emite um ramo que de acordo 
MENEZES, L.T. et al. Comportamento anatômico das artérias da base do encéfalo de javali (Sus scrofa scrofa - Linnaeus, 1758). PUBVET, Londrina, V. 5, N. 13, Ed. 160, Art. 1085, 2011.

com vários autores é a artéria condilar, continuando a artéria carótida interna com o mesmo nome até a rede admirável epidural rostral, que a partir desta estrutura ocorre a emergência de artérias para o encéfalo. No entanto adotamos estes vasos como artéria carótida do encéfalo.

Nickel; Schummer; Seiferle (1981) denominaram o orifício por onde passa a rede admirável de forame carótido, optamos por forame lácero. Schwarze e Schröder (1972) observaram que nos suínos adultos, a porção distal da artéria carótida interna está atrofiada, enquanto que a partir de sua porção proximal, nasce um tronco comum com a artéria occipital, se origina a artéria condílica e artéria carótida interna. Na presente pesquisa adotamos que a artéria carótida interna deve ser assim ser considerada desde a sua origem em tronco comum com a artéria occipital, tendo como ramo a artéria condilar.

Conforme Getty (1986) à artéria carótida interna, normalmente surge por um tronco comum com a artéria occipital, a pesquisa em questão sobre javali também presenciou esta afirmativa acima citada, ou seja, em todas as peças ocorreu esta situação.

Portanto, notou-se que a denominação da artéria carótida interna para este vaso se mostrou imprópria, e identificada por nós pela denominação de artéria carótida do encéfalo com seus ramos.

No tocante ao maior desenvolvimento da artéria carótida interna em embriões, observamos que esta se apresentava desenvolvida também em neonatos de javali, concordando com as afirmações de De Vriese (1905) sobre a artéria carótida interna de embriões. No entanto também foram observadas em peças adultas de javali este desenvolvimento da artéria em questão, sendo que a mesma não se apresentava atrofiada.

No que tange aos vasos terminais da artéria carótida do encéfalo em ambos antímeros, temos que os autores consultados referem-se à existência de dois ramos, que denominamos de ramo rostral e caudal da artéria carótida do encéfalo, definimos estes ramos da artéria carótida pertencente ao encéfalo, pois, a artéria cerebral caudal emite ramos para o mesencéfalo; diferentemente de Nickel; Schummer; Seiferle (1975) que padronizam de 
MENEZES, L.T. et al. Comportamento anatômico das artérias da base do encéfalo de javali (Sus scrofa scrofa - Linnaeus, 1758). PUBVET, Londrina, V. 5, N. 13, Ed. 160, Art. 1085, 2011.

artéria carótida cerebral, sendo dividida em artéria cerebral rostral e comunicante caudal.

Nas citações de Ellenberger e Baum (1943) sobre a artéria coroídea rostral, o mesmo denomina que este vaso e a artéria coroídea nasal, porém de acordo com os achados do trabalho presente, temos que algumas ressalvas em relação a artéria coroídea rostral, pois esta na maioria dos casos se apresentou como originária do ramo rostral da artéria carótida do encéfalo. Já para Nanda (1986) a artéria coroidea tem origem na artéria carótida do encéfalo, divergindo do artigo em questão e de Ferreira, C. (1998) sobre a origem, pois notamos que esta se origina do ramo rostral da artéria carótida do encéfalo e não da artéria carótida interna.

Em relação a artéria cerebral média, Baptista (1922), Gillilan (1974, 1984), Nanda (1986) citam que a artéria média do cérebro de suínos surgem da artéria carótida interna tendo de dois a três ramos, sendo denominados de artérias médias do cérebro. Entretanto Câmara Filho et al. (2004), Ferreira, C. (1998), e o trabalho presente descrevem que a artéria cerebral média é originária do ramo rostral da artéria carótida do encéfalo, tendo número variado de ramos

Nanda (1986) descreve a artéria cerebral rostral é originada da artéria carótida interna, correndo rostralmente no plano mediano ao longo da superfície ventral do trato olfatório medial e da fissura longitudinal do cérebro, entretanto o estudo sobre as artérias da base do encéfalo de javali e Ferreira, C. (1998) e Câmara Filho et al. (2004) descrevem que a artéria cerebral rostral emergem do ramo rostral da artéria carótida do encéfalo, margeando a fissura longitudinal do cérebro penetrando ao nível desta fissura.

Desta maneira percebe-se que as observações deste estudo conferiram com o citado por De Vriese (1905), que considerou ser a artéria cerebral rostral o único ramo terminal da artéria carótida do encéfalo.

Nos informes de Ellenberger e Baum (1943) e Schwarze e Schröder (1972) em relação a artéria etmoidal interna, temos que esta seria originária da artéria cerebral rostral que se encaminharia para a rede etmoidal. Porém 
MENEZES, L.T. et al. Comportamento anatômico das artérias da base do encéfalo de javali (Sus scrofa scrofa - Linnaeus, 1758). PUBVET, Londrina, V. 5, N. 13, Ed. 160, Art. 1085, 2011.

para a pesquisa presente temos que a artéria etmoidal interna tem emergência do ramo rostral da artéria carótida do encéfalo, antes da fusão com a homônima do antímero oposto, caminhando em direção à parte rostral do bulbo olfatório atingindo a lâmina crivosa do etmoíde, formando a rede etmoidal, assim como descrita por Ferreira, C. (1998) e Câmara Filho et al. (2004) em suínos.

Porém Nanda (1986) descreve que a artéria etmoidal interna é continuação rostral da artéria rostral do cérebro após a emissão da artéria comum do corpo caloso, ou seja, divergindo do trabalho presente em relação a origem da artéria em questão.

Relativamente a origem da artéria cerebral caudal, temos que é um ponto importante para o estabelecimento da classificação dos vasos arteriais, no entanto consideramos a sua origem no ramo caudal da artéria carótida interna do encéfalo ou em ramo terminal da artéria basilar, nossos achados corroboram com Lima et al. (2010) nos gatos, a artéria cerebral caudal é originada do ramo caudal da artéria carótida interna; com Alcântara et al. (2000), Nanda (1986) e Lima et al. (2006). E divergem das informações de Câmara Filho et al. (2004) e Ferreira, C. (1998) que comenta a origem desta artéria, estando entre o limite do ramo caudal da artéria carótida do encéfalo e o correspondente ramo terminada da artéria basilar.

No condizente ao comportamento da artéria basilar, o presente estudo mostrou que este vaso arterial é único resultante da união das artérias occipitais em ambos antímeros na transição da medula espinhal com o terço caudal do bulbo assim como demonstrado por Câmara Filho et al. (2004), Ferreira, C. (1998) entretanto divergindo com as informações de Getty (1986), pois este relata a formação da artéria cerebro-espinhal como sendo dividida em ramo basilar e ramo espinhal ventral que se unem a ramos semelhantes do lado oposto para formarem rostralmente a artéria basilar e a artéria espinhal ventral. Guardando ressalvas também em relação as informações de Gillilan (1974) no qual o autor comenta que a artéria basilar no suíno afunila-se posteriormente ao passar pelo tronco cerebral, tornando-se delgada ao passar 
MENEZES, L.T. et al. Comportamento anatômico das artérias da base do encéfalo de javali (Sus scrofa scrofa - Linnaeus, 1758). PUBVET, Londrina, V. 5, N. 13, Ed. 160, Art. 1085, 2011.

pelo cordão espinhal, para a pesquisa em torno da artéria basilar em javali, constatou-se que esta artéria afunila-se na maioria dos casos a partir da artéria cerebelar caudal. Discordamos das informações de Lima et al. (2005) também em relação a origem da artéria basilar pois para este a artéria em questão seria originária das redes epidurais caudais de cada antímero, as quais emitiram um ramo no qual perfuraria a duramater e anastomosaria com a rede contralateral e com a artéria espinhal ventral.

Em relação aos ramos terminais da artéria basilar, notamos que são poucas as referências em relação a este ramo, evidenciamos nas peças analisadas que teriamos duas situações a considerar; primeiro em relação ao calibre da artéria basilar, que nestes animais apresentou-se como um ramo delgado a partir da artéria cerebelar caudal, sendo que a partir deste vaso ocorreria o aparecimento de um ramo para cada antímero, pois se originariam do ramo caudal da artéria carótida do encéfalo, este ramo foi para nós denominado de ramo ponte mesencefálico. Porém se considerarmos a direção da artéria basilar teriamos a presença do ramo terminal da artéria basilar até sua união com o ramo caudal da artéria carótida do encéfalo. Pela as análises realizadas é notório que o javali não apresenta um comportamento definido sobre um modelo arterial do encéfalo, evidenciando que não se encaixa em nenhum dos modelos arteriais propostos por De Vriese (1905) em referência as observações de Testut (1911) e Ferreira, C. e Prada, I. (2005), porém se aproxima do modelo tipo 2 alfa definido pelo autor acima citado.

Diferentemente das informações obtidas por Gillilan (1984) a respeito dos ramos da artéria basilar, onde, este autor define como artéria cerebelar inferior e artéria cerebelar superior, no qual a primeira teria destino na superfície caudal do cerebelo e a segunda seria os primeiros ramos da artéria basilar e se distribuiriam na porção dorsal e rostral do cerebelo pois, em javali utilizamos os termos para os vasos de artéria cerebelar caudal tendo emergência da artéria basilar, se dirigindo para a porção caudal do cerebelo e artéria cerebelar rostral onde irrigaria a porção rostral do cerebelo. Para a pesquisa em questão denominamos de artéria cerebelar caudal e artéria 
MENEZES, L.T. et al. Comportamento anatômico das artérias da base do encéfalo de javali (Sus scrofa scrofa - Linnaeus, 1758). PUBVET, Londrina, V. 5, N. 13, Ed. 160, Art. 1085, 2011.

cerebelar rostral. Já para Câmara Filho et al. (2004) e Nanda (1986) a artéria cerebelar caudal e artéria cerebelar rostral seriam ramos da basilar, no entanto Nanda (1986) ainda acrescenta que a primeira teria uma direção que atingiria a superfície dorsal da medula oblonga e terminariam em dividir-se em três ramos, e que esta emitiria a artéria média do cerebelo; a segunda seria um ramo da artéria mesencefálica. Divergindo dos autores acima a pesquisa presente evidenciou que a artéria cerebelar rostral teria origem no ramo terminal da artéria basilar ou na artéria basilar, mostrando que este animal não definiu um modelo para o arranjo vascular destas artérias.

\section{CONCLUSÕES}

Em relação ao comportamento das artérias da base do encéfalo de javali, julgamos poder concluir que:

- As artérias da base do encéfalo estão na dependência da artéria carótida do encéfalo em ambos os antímeros e da artéria basilar e seus ramos;

- A rede admirável epidural rostral se origina a artéria carótida do encéfalo, constituída com a participação das artérias carótida interna e maxilar interna e seus ramos, artérias meníngea média e oftálmica externa;

. A artéria carótida do encéfalo apresenta um ramo rostral e um ramo caudal;

- A artéria cerebral caudal originou-se em alguns modelos do ramo caudal da artéria carótida do encéfalo e em alguns modelos do ramo terminal da artéria basilar;

. Não existe padrão para as artérias da base do encéfalo deste animal, porém tende seguir o padrão vascular tipo 2 alfa de De Vriese (1905).

\section{REFERÊNCIAS}

ALCÂNTARA, M. A. Estudo anatômico das artérias da base do encéfalo de cães (Canis familiaris, LINNAEUS, 1758). São Paulo: USP, 1992. 101f. Dissertação (Mestrado em Anatomia de animais Domésticos e Silvestres) - Pós- graduação em Anatomia de animais 
MENEZES, L.T. et al. Comportamento anatômico das artérias da base do encéfalo de javali (Sus scrofa scrofa - Linnaeus, 1758). PUBVET, Londrina, V. 5, N. 13, Ed. 160, Art. 1085, 2011.

Domésticos e Silvestres, Faculdade de Medicina Veterinária e Zootecnia, Universidade de São Paulo, São Paulo, 1992.

ALCÂNTARA, M. A.; ALMEIDA, I. C.; MICHAELKI, F. Z. A Artéria cerebral caudal em cães (Canis familiaris, Linnaeus 1758). SRD - estudo anatômico de seus segmentos e distribuição. Ciência Cultura, Tuiuti, v.21, n. 21, p. 57-69, Mar. 2000.

BARBOSA, L. L.; PURRIEL, L. A; MEERHOFF, W.; MEDOC, I. El polígono de Willis y sus variaciones. Acta Neurologica Latinoamericana, Montevideo, v. 15, n.3, p.224-36, Jan. 1969.

BAPTISTA, B. V. Estudo comparado da circulação cerebral dos mamíferos domésticos e no homem - Razão de ser da rede admirável. Rio de Janeiro: UFRJ, 1922. 89f. Tese (Doutorado em Anatomia) - Pós-graduação em Anatomia, Faculdade de Medicina, Universidade Federal do Rio de Janeiro, Rio de Janeiro, 1922.

BRUNI, A. C.; ZIMMERL, U. Anatomia degli animali domestici. Milano: Francesco vallardi, 1947. v.2. 319-324p.

CÂMARA FILHO, J. A.; SCHERER, P. O.; SCHERER, R. R.; MENEZES, C. M. C. Características morfológicas da distribuição vascular cerebral de Sus scrofa Linnaeus (Mammalia, Artiodactyla). Revista Brasileira de Zoologia, São Paulo, v. 21, n.4, p.955-959, Dez. 2004.

CAMPOS, A.; PRADA, I. L.S; SANTOS JUNIOR, I.; SANTOS, D. Artérias da base do encéfalo de eqüinos. Sistema occipito-basilar. Brazilian Journal of Veterinary Research and Animal Science, São Paulo. v.40, n.1, p.107-117, Abr. 2003.

CROSBY, E. C.; SCHNITZLRIN, H. N. Comparative correlative neuroanatomy of the vertebrate telencephalon. New York: Mac Millan, 1982, p. 301-309.

DYCE, K. M.; SACK, W. O.; WENSING, C. J. G. Tratado de anatomia veterinária. Rio de Janeiro: Guanabara Koogan, 1990. 163p.

ELLENBERGER, W.; BAUM, H. Handbuch der vergleichenden anatomie der haustiere. Berlim: Springer-Verlag, 1943. 669-671p.

FERREIRA, C. G. Estudo anatômico das artérias da base do encéfalo de suínos (Sus scrofa domesticus-LINNAEUS-1758). São Paulo: USP, 1998. 98f. Tese (Doutorado em Anatomia de animais Domésticos e Silvestres) - Pós- graduação em Anatomia de animais Domésticos e Silvestres, Faculdade de Medicina Veterinária e Zootecnia, Universidade de São Paulo, São Paulo, 1998.

FERREIRA, C. G.; PRADA, I. L. S. O circuito arterial da base do encéfalo em suínos (Sus scrofa domesticus, Linnaeus, 1758), formação e comportamento. Brazilian Journal Veterinary Research Animal and Science, São Paulo, v.42, n.1, p.53-60, Mar. 2005.

FERREIRA, J. R.; PRADA, I. L. S. O sistema carótico do encéfalo do Cebus apella sp., Linnaeus, 1766. Biota Neotropica, São Paulo, v.9, n.1, p.285-295, Mar. 2009. Disponível em:

$<$ http://www.biotaneotropica.org.br/v9n1/pt/abstract?shortcommunication+bn03209012009 > Acesso em: 24 jan. 2011.

FERREIRA, J. R. Estudo anatômico das artérias da base do encéfalo do macaco prego (Cebus apella, LINNAEUS, 1766). São Paulo: USP, 1997. Tese (Doutorado em Anatomia de animais Domésticos e Silvestres) - Pós- graduação em Anatomia de animais Domésticos e 
MENEZES, L.T. et al. Comportamento anatômico das artérias da base do encéfalo de javali (Sus scrofa scrofa - Linnaeus, 1758). PUBVET, Londrina, V. 5, N. 13, Ed. 160, Art. 1085, 2011.

Silvestres, Faculdade de Medicina Veterinária e Zootecnia, Universidade de São Paulo, São Paulo, 1997.

FLECHSIG, G.; ZINTZSCH, I. Die arterien der schadelbasis der schweines. Anatomischer Anzeiger, German, v.125, n.5, p.206-219, Jun. 1969.

GETTY, R. Sisson/Grossman Anatomia dos animais domésticos. Rio de Janeiro: Guanabara Koogan, 6.ed, v.2, 1986. 1230-1237p.

GILLILAN, L. A. Blood supply to brains of ungulates with and without a rete mirabile caroticum. The Journal of comparative neurology, New York, v.153, n.3, p.275-90, Jun. 1974.

GILLILAN, L. A. Blood supply of vertebrate brains. In: CROSBY, E. C.; SCHNITZLRIN, H. N. Comparative neuroanatomy of the vertebrate telencephalon. New York: Mac Millan, cap.6, 1984. p.266-373.

GONZALEZ Y GARCIA, J.; GONZALEZ ALVAREZ, R. Anatomia comparada de los animales domesticos. Zaragoza: Canalez, 1929. p.678-82.

INTERNATIONAL COMMITTEE ON VETERINARY GROSS ANATOMICAL NOMENCLATURE. Nomina anatomica veterinaria. 5ed. Columbia: Editorial Committee Hannover, 2005.

LIMA, E. M. M.; SEVERINO, R.S.; DRUMMOND, F. O. S. S.; BOMBONATO, P. P.; RODRIGUES, D. B. Artérias da Base do Encéfalo em suínos Camborough 22. Bioscience Journal, Uberlândia, v.21, n.2, p.137-147, May/Aug. 2005.

LIMA, E. M. M.; PRADA, I. L. S.; SILVA, F. O. C.; SEVERINO, R. S.; SANTOS, A. L. Q.; DRUMMOND, S. S.; RODRIGUES, G. S. Estudo anatômico das artérias da base do encéfalo em gatos. Ars Veterinaria, Jaboticabal, v. 22, n.1, p. 001-007, Jan. 2006.

LIMA, E. M. M.; PRADA, I. L. S.; SILVA, F. O. C.; SEVERINO, R. S.; SANTOS, A. L. Q.; BORGES, B. O.; PAIM, T. P.; VIANNA, A. R. C. B. Sistematização da origem, da distribuição e dos territórios da artéria cerebral caudal na superfície do encéfalo em gatos. Ciência Rural, Santa Maria, v.40, n.9, p.1961-1965, Set, 2010.

LINDERMANN, T. Sistematização da irrigação na base do encéfalo do gambá (Didelphis albiventris). Porto alegre: UFRGS, 1994. 122p. Dissertação (Mestrado em Anatomia dos Animais Domésticos) - Pós-graduação em Anatomia dos Animais Domésticos, Faculdade de Veterinária, Universidade Federal do Rio Grande do Sul, Porto Alegre, 1994.

LUI, J. F. Estudo citogenético de javalis puros (Sus scrofa scrofa)e híbridos nas regiões sudeste e sul do Brasil. Revista de educação continuada CRMV-SP, São Paulo, v.3, fascículo 1, p 43-48. 2000.

MANNU, A. Apparechio vascolare. In: Zimmerl, V. Trattado di anatomia veterinaria. Milan: Francesco Vallardi, v.2. 1930. p.79-99

MELO, A. P. F. Estudo anatômico das artérias da base do encéfalo em fetos de bovinos azebuados. São Paulo: USP, 1996. 245p. Dissertação (Mestrado em Anatomia de animais Domésticos e Silvestres) - Pós- graduação em Anatomia de animais Domésticos e Silvestres, Faculdade de Medicina Veterinária e Zootecnia, Universidade de São Paulo, São Paulo, 1996.

MONTANÉ, L.; BOURDELLE, E. Anatomie regionale des animaux domestiques. Paris: J. B. Baillière, v.2, 1920. p.58-9. 
MENEZES, L.T. et al. Comportamento anatômico das artérias da base do encéfalo de javali (Sus scrofa scrofa - Linnaeus, 1758). PUBVET, Londrina, V. 5, N. 13, Ed. 160, Art. 1085, 2011.

NANDA, B. S. Suprimento sanguíneo para o cérebro. In: GETTY, R. Sisson/Grossman Anatomia dos animais domésticos. Rio de Janeiro: Guanabara Koogan, 6.ed, v.2, 1986. 1232-1237p.

NICKEL, R.; SCHUMMER, A.; SEIFERLE, E. Nervensystem Sinnesorgane endokrine Drusen. Berlim: Paul Parey, v.4. 1975. p.174-181.

NICKEL, R.; SCHUMMER, A.; SEIFERLE, E. The circulatory system, the skin, and the cutaneons organs of the domestic mammals. Berlim: Paul Parey. v.3. 1981. P.101-105.

RECKZIEGEL, S. H. Sistematização da irrigação na base do encéfalo de capivara (Hydrochoerus hydrochoeris). Porto alegre: UFRGS, 1994. 137p. Dissertação (Mestrado em Anatomia dos Animais Domésticos) - Pós-graduação em Anatomia dos Animais Domésticos, Faculdade de Veterinária, Universidade Federal do Rio Grande do Sul, Porto Alegre, 1994.

SANTOS, R. M. B. Estudo anatômico das artérias da base do encéfalo em ovinos (Ovis aries, L., 1758). São Paulo: USP, 1993. 45p. Tese (Doutorado em Anatomia de animais Domésticos e Silvestres) - Pós- graduação em Anatomia de animais Domésticos e Silvestres, Faculdade de Medicina Veterinária e Zootecnia, Universidade de São Paulo, São Paulo, 1993.

SCHWARZE, E.; SCHRÖDER, L. Compêndio de anatomia veterinária. Zaragoza: Acríbia. v.3, 1972. 53-56p.

SCREMIN, O. N. Cerebral vascular sytem. In: PAXINON, G. The rat nervous sytem. New York: Academic Oress, 1995. p.3.

SIMOENS, P.; LAWERS, H.; DE GEEST, J. P.; DE SCHAEPDRIJVEER, L. Functional morphology of the cranial retia mirabilia in the domestic mammals. Schweizer Archiv für Tierheilkunde, Zurich, v.129, n.9, p.295-307, Sept. 1987. 